\title{
Améliorations attendues avec les nouvelles technologies PV : rendements, coûts et cycle de vie
}

"Cet article pour la revue J3EA, a fait l'objet d'une communication aux journées 2007 de la section électrotechnique sur le thème Energie et Développement Durable à l'antenne de Bretagne de l'ENS de Cachan les 14 et 15 mars 2007."

\author{
Jean-Claude Muller
}

InESS (UMR 7163, Laboratoire Commun CNRS - ULP), 23 rue du Loess, B.P.20 F-67037 Strasbourg cedex (France)

\begin{abstract}
Résumé - Le secteur des composants opto-électroniques qui se développe le plus rapidement est sans conteste, celui du photovoltaïque (PV) pour une production d'électricité basée sur la conversion de la lumière du soleil. Pour le moment, plus de $90 \%$ des cellules sont encore à base de silicium cristallin, qui est la voie la plus avancée sur le plan technologique et industriel avec une durée de vie garantie des modules PV de plus de 25 ans. En effet, le silicium est l'un des éléments les plus abondants sur terre, parfaitement stable et non toxique. Dans un avenir proche, d'autres matériaux au stade préindustriel comme certains matériaux en couches minces à base de silicium ou de chalcogénures et à une échéance plus lointaine, des matériaux encore au niveau de la recherche comme les cellules organiques à base de polymères et d'autres au niveau de la validation des concepts viendront compléter l'offre de produits photovoltaïques et contribuer à la stimulation de ce marché très prometteur.
\end{abstract}

Mots clés : composants opto-électroniques, semiconducteurs, matériaux photovoltaïques, silicium, chalcogénures, matériaux organiques.

\section{INTRODUCTION}

La production industrielle mondiale de modules photovoltaïques est 20 fois plus importante qu'il y a 10 ans et a dépassée les $2 \mathrm{GWc}$ en 2006. Pourtant, malgré l'immense chemin parcouru, l'électricité solaire n'a pas encore franchi le seuil lui permettant d'être compétitif par rapport aux autres sources de production d'électricité. Il reste un effort d'un facteur 3 à obtenir pour le coût du Watt installé ainsi que pour le coût du kWh produit.

Ceci nécessitera de nouvelles approches technologiques pour toutes les filières de la plus ancienne et la plus industrialisée comme le silicium cristallin, aux nouveaux matériaux en couches minces, afin de réduire les coûts de production tout en augmentant le rendement de conversion (voir figure 1). De plus, il reste encore deux verrous technologiques à briser tels qu'une fabrication des cellules moins gourmande en énergie et sans répercussion sur l'environnement

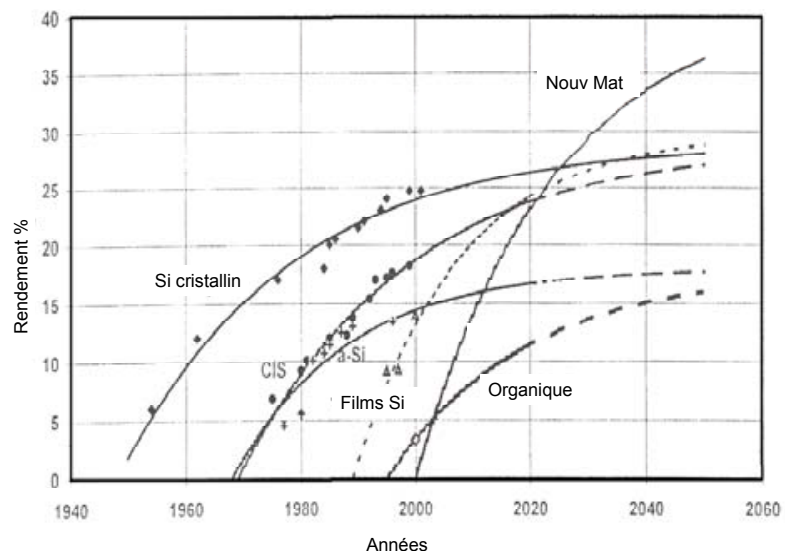

Fig.1. Evolution des rendements des cellules photovoltaïques : à base de silicium cristallin, de couches minces de silicium (amorphe ou polycristallin), de chalcogénures (CIS) et de matériaux organiques ainsi que les nouveaux concepts [1].

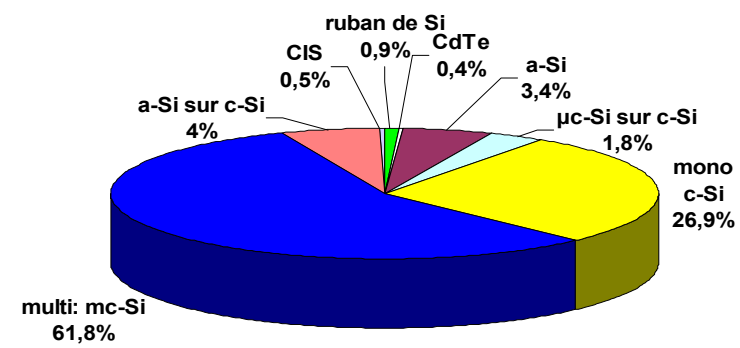

Fig. 2 Illustration de la prépondérance des matériaux à base de silicium cristallin dans la production de cellules [2]

La production de cellules à base de silicium massif multicristallin représente actuellement plus de $60 \%$ des ventes avec encore $27 \%$ en silicium monocristallin. Le silicium amorphe (a-Si) est tombé à près de $3 \%$ du marché et est dépassé par la montée en puissance des hétérojonctions à base de a-Si en couche mince sur du silicium cristallin (4\% du marché). 
La filière silicium cristallin évolue pour des questions de coût et de budget thermique vers des techniques de croissance de plus en plus rapides (coulée continue) et du silicium charge de moins en moins purifié (silicium de qualité solaire, les rebuts de la micro-électronique étant actuellement trop limités).

\section{CEllule a baSe De SILICIUM CRISTAllin MASSIF}

\section{A. Silicium charge}

Le silicium, à la base de toute l'industrie électronique moderne, est obtenu par réduction de la silice dans un four électrique, ce qui fournit le matériau dit "métallurgique" dont la pureté est de $98 \%$ environ. Celui-ci est purifié à son tour, sous forme de gaz trichlorosilane ou silane. Après pyrolyse de ces derniers, le matériau obtenu sert de produit de départ pour la croissance. Cette matière première coûte de plus en plus chère et est passé en quelques années de $15 \$$ à des prix de l'ordre de $150 \$ / \mathrm{kg}$ en raison de la forte augmentation de la demande, de ce fait de grands groupes REC en Norvège, en France (projet Photosil) et Kawasaki Steel au Japon [3] se préoccupent de développer un matériau de qualité solaire avec des techniques de purification simplifiées.

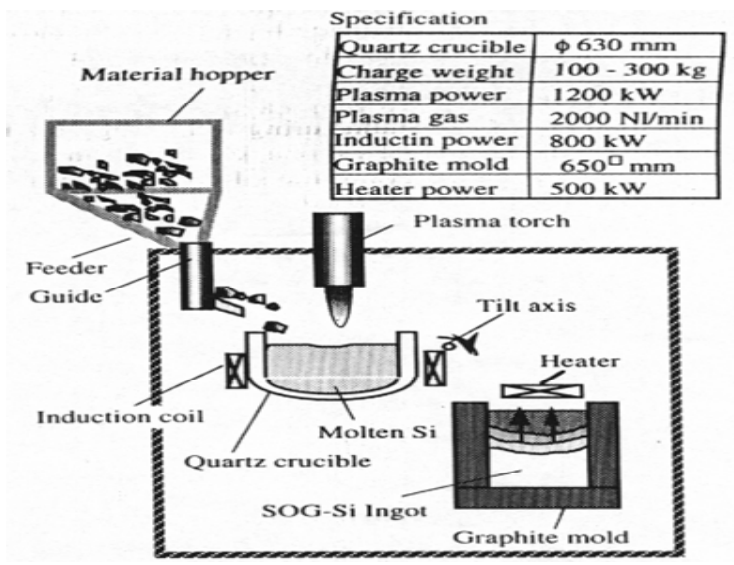

Fig. 3. Techniques spécifiques de purification du silicium de qualité solaire pour le photovoltaïque [3].

\section{B. Silicium monocristallin et multicristallin}

\section{1) Etat de l'art}

Avec les matériaux Czochralski $(\mathrm{Cz})$ et par zone flottante (FZ) de la micro-électronique, il est possible d'obtenir des rendements de conversion de plus de $22 \%$ en laboratoire avec du matériau $\mathrm{Cz}$ (FhG-ISE, Allemagne) et le record de $24,7 \%$ est détenu par l'Université de New South Walles, Australie pour une cellule sophistiquée (voir figure 4) réalisée sur un matériau FZ [4] avec une double couche antireflets, une texturation en pyramides inversées, un émetteur sélectif et un champ arrière repousseur d'électrons (BSF).

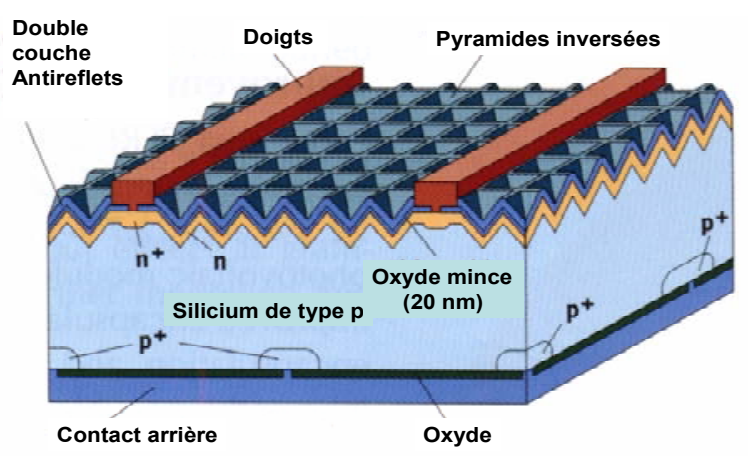

Fig.4. Structure de la cellule de laboratoire la plus performante. Source Photovoltaics Special Research Center UNSW [4].

\section{2) Techniques de croissance des multicristaux}

Par contre, dans un polycristal, les monocristaux sont séparés les uns des autres par des zones perturbées, les joints de grains et chaque cristal de plus petite dimension n'a pas la même orientation que son voisin. On privilégiera les techniques de croissance qui assurent la formation d'une structure colonnaire avec de gros cristaux (d'où l'appellation silicium multicristallin, mc-Si) afin de limiter les effets néfastes des joints de grains.

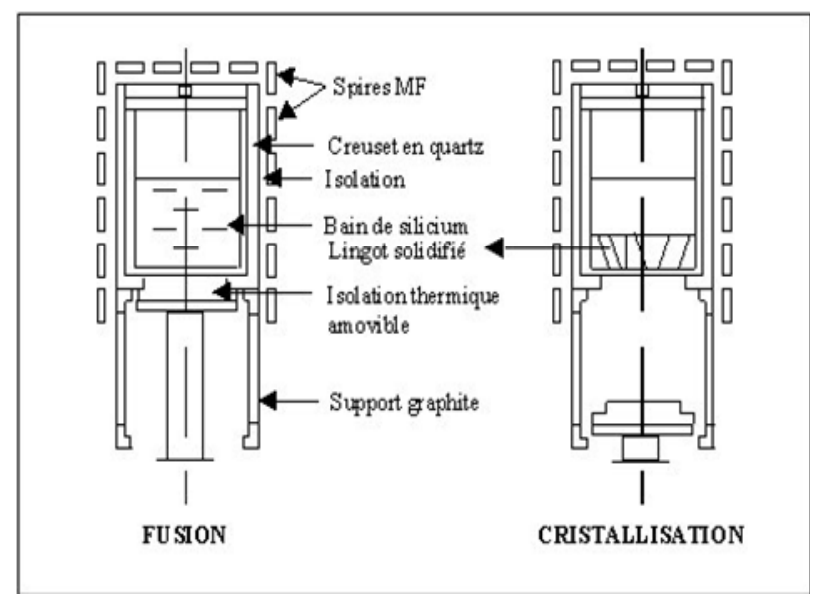

Fig.5. Principe de croissance par solidification directionnelle du type Polix [5] développé par Photowatt S.A.

Cette technique par solidification directionnelle, où des blocs de $240 \mathrm{~kg}$ peuvent être obtenus par refroidissement contrôlé du silicium en fusion dans un moule de nature appropriée (souvent en quartz), est plus rapide et moins coûteuse en énergie qu'une croissance d'un lingot $\mathrm{Cz}$ ou FZ ( 24 à $48 \mathrm{~h}$ contre quelques semaines pour ces derniers). 


\section{3) Applications industrielles}

Cependant ce matériau contraint et disloqué est également contaminé par des impuretés résiduelles provenant de la charge de silicium qui est constituée par un mélange de matière première et de rebuts de l'industrie $\mathrm{PV}$ ou microélectronique. Les premiers matériaux mc-Si ont été le Silso de Wacker en Allemagne dès 1975 [6], suivi en France par le Polix de Photowatt. Aux Etats-Unis, les matériaux les plus connus sont le Semix de Solarex (devenu BP-Solar) et le HEM de Crystal Systems.



Fig. 6. Principales étapes de fabrication du matériau, de la cellule et du module de la Sté Photowatt. S.A. FR [7]

Les Japonais proposent depuis les années 90 un nouveau matériau produit par coulée continue en creuset électromagnétique (Osaka Titanium Co. (OTC) devenu le Sitix de Summitomo [8]). En France, le LPM-Madylam de Grenoble [9] a exploré cette possibilité de tirage en creuset froid (Emix) et a donné lieu à la création de la Sté EMIX [10] en janvier 2004 (La Souterraine, Creuse).

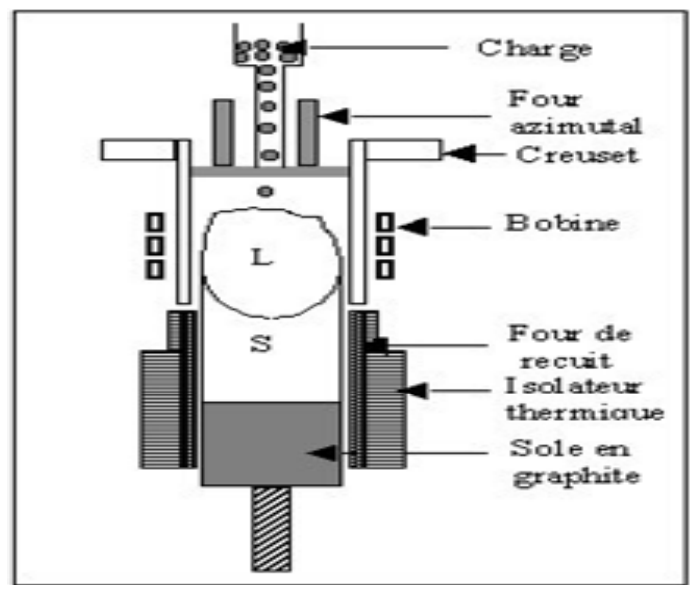

Fig. 7. Principe de croissance de silicium par coulée continue en creuset électromagnétique. Source Madylam \&Emix FR [9].

\section{4) Perspectives}

Les rendements de conversion industriels, qui étaient de l'ordre de 8 à $10 \%$ avant 1980, sont actuellement de 16 à $17 \%$ pour de grandes plaquettes de $10 \times 10$ ou $15 \times 15 \mathrm{~cm}^{2}$. Ces progrès s'expliquent par l'amélioration constante de la qualité des matériaux et par une réduction de l'activité électrique des impuretés résiduelles.

Depuis l'augmentation des coûts de la matière première de silicium, les industriels s'orientent vers l'utilisation de plaquettes de plus en plus minces (de $300 \mu \mathrm{m}$ à moins de $150 \mu \mathrm{m})$ ce qui nécessite une évolution des dispositifs industriels avec déjà une utilisation systématique du champ arrière (BSF) et probablement à l'avenir vers des structures à contacts interdigités uniquement en face arrière (RCC).

Toutefois, le sciage de ces grands blocs reste une opération onéreuse conduisant à une perte non négligeable de matière, d'où les alternatives qui consistent à recourir à du silicium pouvant être mis en oeuvre sans recourir à des opérations de découpe.

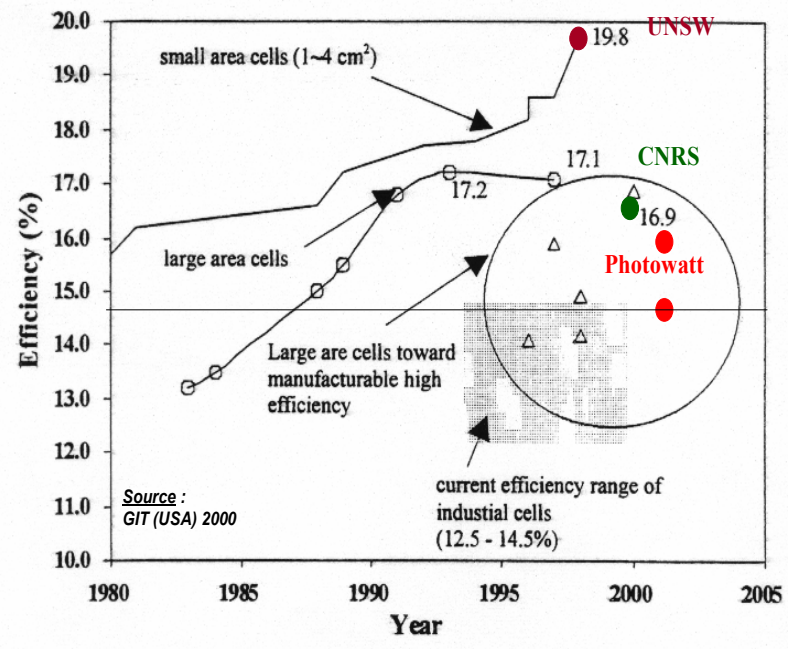

Fig.8. Progression des rendements de conversion en laboratoire et dans industrie des cellules [11].

\section{Silicium en ruban autosupporté}

\section{1) Etat de l'art}

La croissance de silicium sous forme de rubans contribue à diminuer les pertes de matière première. Ceux-ci ont connus, dans les années 90, de nombreux développements au niveau de la recherche et pour certains d'entre eux jusqu'à la conception de chaînes de production préindustrielles. Les rendements de conversion des rubans (de l'ordre de 14 à $15 \%$ ) sont systématiquement inférieurs à ceux obtenus pour les matériaux cristallins en raison des contraintes spécifiques de leur croissance cristalline. 


\section{2) Techniques de croissance}

Les techniques de production de silicium en rubans autosupportés ont été très séduisantes sur le plan technologique, qu'ils soient obtenus par capillarité entre deux lèvres de carbone ou par croissance sur un film ou une maille de carbone.

Cependant, toutes ces techniques "ruban" ont toujours été largement pénalisées par une vitesse de croissance linéaire (généralement de quelques $\mathrm{cm} / \mathrm{min}$ à quelques dizaines de $\mathrm{cm} / \mathrm{min}$ ), imposée par les critères de solidification qui déterminent la taille des grains et la pureté du matériau par la ségrégation des impuretés.

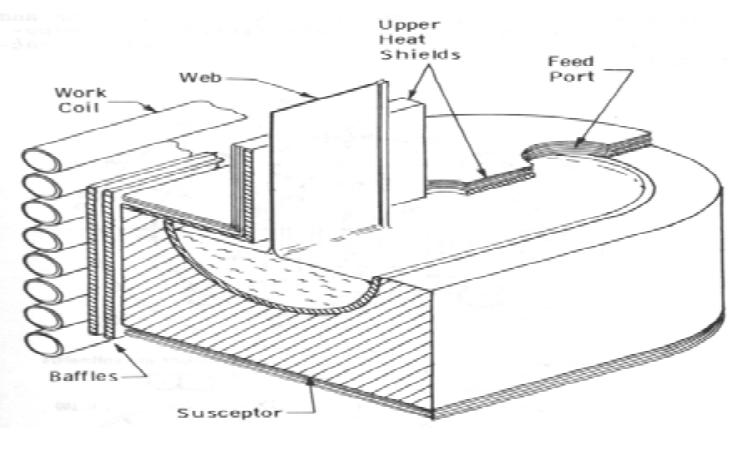

Fig. 9. Procédé de croissance de silicium en ruban du type WEB USA [ 12].

\section{3) Applications industrielles}

Les raisons évoqués ci-dessus ont pratiquement marginalisé la production de rubans $(1 \%$ de la production mondiale), sauf aux Etats Unis ou les rubans du type EFG de la Société Mobil Solar (actuellement RWE-Schott Solar) subsistent sous forme d'octogones de silicium afin augmenter la capacité de production.

\section{4) Perspectives}

Des industriels tel que Evergreen Solar aux USA et Solarforce en France misent sur une matière premire de silicium qui restera élevé et proposent des techniques permettant une réduction importante de l'épaisseur du ruban $(<100 \mu \mathrm{m})$.

\section{Cellules en COUCheS Minces}

\section{A. Silicium polycristallin}

La technologie en couches minces de silicium cristallin déposé sur un support, dont le développement sera largement déterminé par le coût du silicium charge, consiste à déposer une couche mince de silicium polycristallin de 10-40 $\mu \mathrm{m}$ d'épaisseur sur divers substrats de silicium métallurgique peu purifié, quartz, céramique ou métal.

Ces couches minces devraient permette d'obtenir des rendements de conversion du même ordre qu'une cellule sur matériau massif, moyennant un confinement optique et un champ arrière répulsif pour les porteurs minoritaires et, du fait que la distance qu'ils ont à parcourir est plus faible, on peut se contenter d'une pureté moindre pour le matériau de base.

\section{1) Etat de l'art}

Les rendements des techniques par dépôt restent cependant inférieurs à $10 \%$ et ne passent à des valeurs de 13-16\% qu'avec une recristallisation pour augmenter la taille des grains. Sur support céramique on est parvenu à un rendement de $9,5 \%$

Voir la figure 10 sur laquelle les rendements de conversion en Laboratoire des cellules à base de silicium polycristallin sont donnés en fonction de la taille des grains.

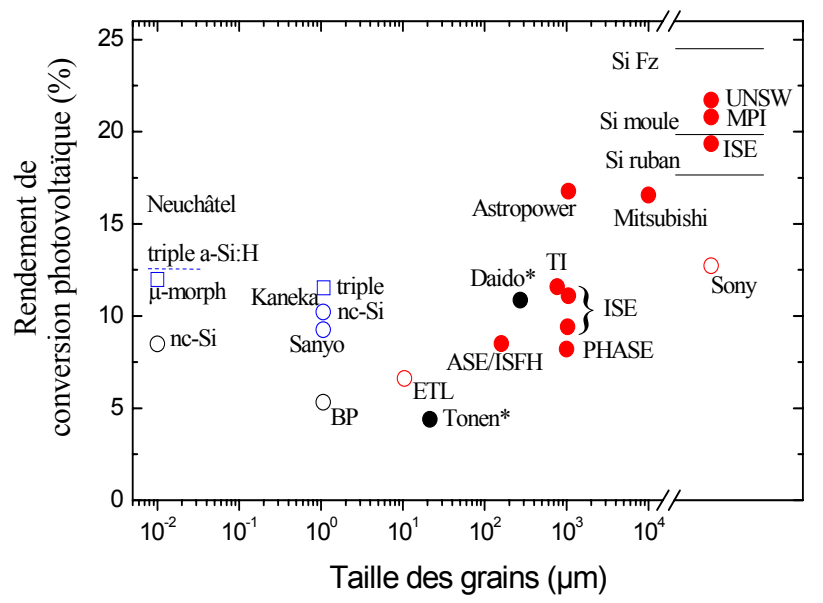

Fig.10. Progression des rendements de conversion en Laboratoire des cellules à base de silicium polycristallin en fonction de la taille des grains.[13].

\section{2) Techniques de dépôt des films minces}

Deux techniques sont actuellement en compétition, la croissance par épitaxie en phase liquide (LPE) dont le principal inconvénient est l'utilisation d'un substrat cristallin (19-21\% de rendement en laboratoire) et les dépôts en phase solide.

Pour ces derniers on citera, la décomposition pyrolytique du silane et de l'hydrogène sur un filament chaud de tungstène (LPICM) permettant de déposer du 
silicium microcristallin $(\mu \mathrm{c}-\mathrm{Si})$ sur un substrat de verre à $500^{\circ} \mathrm{C}$ à des vitesses relativement élevées $(>5 \mu \mathrm{m} / \mathrm{h})$, les dépôts en phase vapeur (CVD) à des températures $>800^{\circ} \mathrm{C}$ en présence d'un gaz contenant le silicium (silane ou chlorosilanes).

Une croissance plus rapide avec des vitesses de dépôts de l'ordre de $10 \mu \mathrm{m} / \mathrm{mn}$ par CVD assistée par lampes (RTCVD) est également possible (CNRS-InESS). Dans ce cas le substrat n'est chauffé que pendant la durée du dépôt à des températures bien plus élevées que celles du gaz.

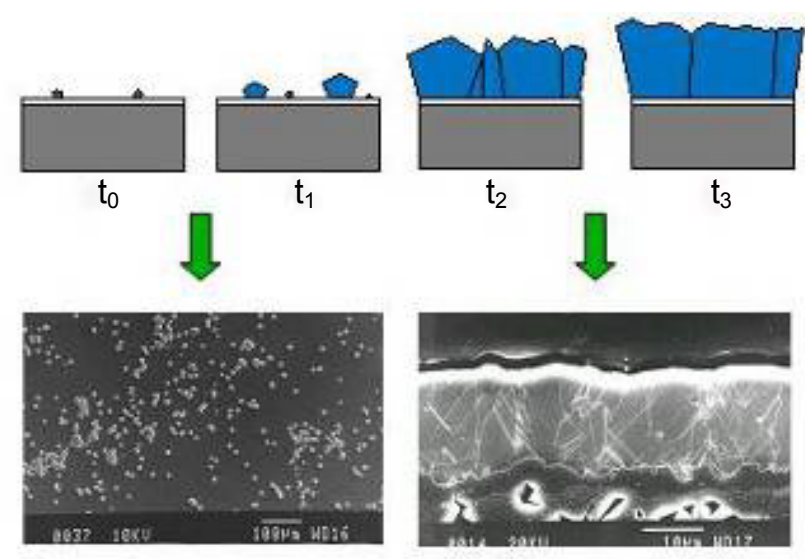

Fig. 11. Croissance de silicium en film mince sur céramique par RT-CVD. Source CNRS-InESS [14 ].



Fig. 12. Procédés de transfert ou de pelage d'une couche mince de silicium par voie poreuse ou ionique $[15,16]$

Les techniques de dépôt rentrent en compétition avec les méthodes de transfert de films de silicium. La première étape consiste à déposer deux couches de silicium macro- et micro-poreux sur du silicium monocristallin et de faire croître par épitaxie une couche de silicium quasimonocristalline sur la couche la moins poreuse.

Les étapes suivantes consistent à terminer la cellule par un dépôt de contact avant de dissoudre la couche la plus poreuse et transférer le tout sur un substrat étranger [15] (voir figure 12 gauche). Un projet analogue a été développé en France par l'INSA de Lyon [16] (figure 12 à droite).

\section{3) Applications industrielles et perspectives}

Depuis quelques années, les films minces de silicium cristallin suscitent un intérêt majeur aux USA et en Europe où CGS Solar a abouti à la première fabrication industrielle de cellules en couches minces performantes.

\section{B) Silicium nanocristallin \& amorphe}

\section{1) Etat de l'art}

Depuis 1975, des recherches intensives ont été entreprises pour utiliser du silicium non cristallisé, à l'état amorphe, dont les liaisons pendantes sont saturées par hydrogénation. Ce matériau présente trois avantages majeurs: un fort coefficient d'absorption ce qui autorise de très faibles épaisseurs l'ordre du micron diminuant ainsi le risque de pénurie de silicium, une faible consommation énergétique durant le cycle de production avec un temps de retour énergétique inférieur à un an, et une aptitude à être déposé en grandes surfaces unitaires de l'ordre $\mathrm{du}^{2} \mathrm{~m}^{2}$ d'un seul tenant.

Par contre ses deux points faibles sont le rendement de conversion et la dégradation sous lumière (instabilité dite de Staebler-Wronski). Ces effets sont de mieux en mieux surmontés par des artifices technologiques tel qu'en particulier par une superposition de deux structures p-i-n en "tandems" ou trois structures $\mathrm{p}$-i-n superposées (voir figure 13). On a réduit ainsi la dégradation sous lumière de $30 \%$ à $10 \%$ et grâces à des couches actives très fines le rendement atteint $13 \%$.

2) Techniques de dépôt du matériau et réalisation de la structure.

La cellule à base de silicium amorphe se compose d'une zone dopée bore, intrinsèque et dopée phosphore ( $p-i-n)$. Elle est obtenue par dépôt sous vide de couches minces de silicium par dissociation d'un plasma de silane ( $\mathrm{SiH} 4)$ en présence d'un gaz dopant tel que le phosphine (PH3) pour la couche $\langle\mathrm{n} »$, de diborare (B2H6) pour la couche $\langle\mathrm{p} »$ et sans apport de gaz pour la couche intrinsèque $« i »$.

Pour cette simple structure industrielle à trois couches (p-i-n), chaque élément peut être directement connecté en série sans besoin de liaisons externes [17], les rendements industriels stagnent depuis des années sous la barre des 10 $\%$, de sorte que des études très prometteuses ont été reprises 
dans le domaine des matériaux nano- ou micro-cristallins et même presque amorphes (micro-morphe) avec des rendements de conversion déjà supérieurs à $10 \%$.

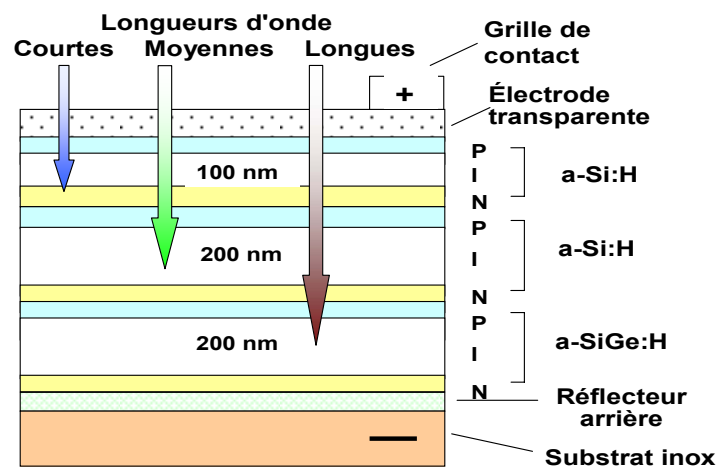

Fig13. Cellule à base de silicium amorphe à triple structure p-i-n,

En effet, ces travaux ont démontré que des couches de $\mu$-cristallines ou $\mu$-morphes de silicium hydrogéné déposées en utilisant des techniques de décharge radio fréquence permettent de réaliser des cellules ne présentent plus aucune dégradation sous exposition à la lumière. L'idée est d'empiler une cellule $\mathrm{p}-\mathrm{i}-\mathrm{n}$ a-Si:H classique avec une cellule extrêmement fine de quelques $\mu$ d'épaisseur du type $\mathrm{p}-\mathrm{i}-\mathrm{n}$ $\mu \mathrm{c}-\mathrm{Si}: \mathrm{H}$ (à l'Institut de microtechnique de Neuchâtel) ou pi-n $\mu$-morphe Si:H (Laboratoire LPICM de l'Ecole Polytechnique de Palaiseau) [18].

\section{3) Applications industrielles}

La toute première commercialisation a concerné le marché des calculettes solaires par Sanyo au début des années 80, puis d'autres applications «grand public » tels que des lampes tempête ou de jardin, des Kits photovoltaïques et systèmes de sécurité. Les améliorations successives du procédé ont contribué à faire passer en 20 ans le rendement industriel des cellules $\mathrm{p}-\mathrm{i}-\mathrm{n}$ non encapsulées de $4 \%$ à un rendement encapsulé de $8 \%$ et plus récemment le développement des jonctions triples dont la dernière est dopée au Germanium a permis d'atteindre des rendements stabilisés de $12 \%$ en laboratoire et de $10 \%$ en pilote industriel.

Si les petits systèmes "grand public" ont été le principal débouché de l'amorphe pendant de nombreuses années, des produits souples (à jonction triple tel que celles proposées par Canon) et des applications pour les bâtiments sont visées depuis la fin des années 1990. Ainsi des tuiles, bardeaux et autres éléments de toitures et de façades incorporant des cellules amorphes ont été développés en coopération avec des professionnels du bâtiment.

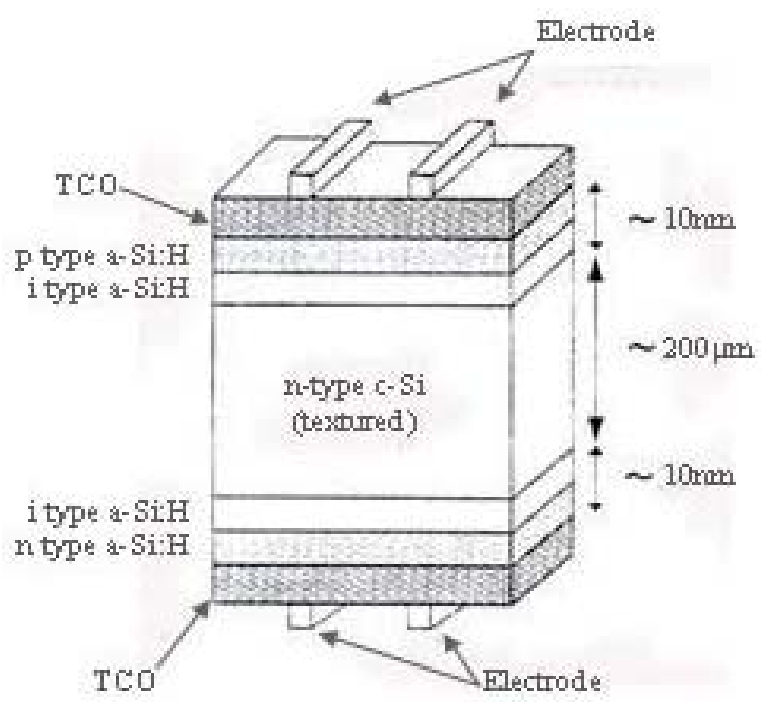

Fig. 14. Hétéro-structure à base de a-Si / silicium cristallin (structure HIT de Sanyo)[19]

\section{4) Perspectives}

Cependant les parts de marché du silicium amorphe sont en chute libre avec à peine plus de $3 \%$ alors qu'il y a 5 ans le a-Si représentait encore $10 \%$ du marché. L'avenir de ces couches passera probablement par un mariage avec le cristallin (voir figure 14).

En effet, les hétéro-structures à base de a-Si / silicium cristallin (structure HIT de Sanyo) présentent une production en forte croissance (de l'ordre de 4\% du marché) avec des rendements de laboratoire de plus de $21 \%$ et $16 \%$ en production industrielle [20].

\section{Autres matériaux en couches minces émergeants}

Les recherches intensives entreprises depuis de nombreuses années sur d'autres matériaux que ceux à base de silicium ont enfin aboutis vers la fin de cette décennie au stade de l'industrialisation. Ils occupent une part encore négligeable du marché (env. 0,9\%).

\section{1) Etat de l'art}

La plupart des cellules au Cd Te utilisent une couche de $\mathrm{CdS}$ de type $\mathrm{n}$ comme fenêtre d'entrée de la lumière et comme barrière de potentiel (hetero-jonction CdS-CdTe). Bien que les derniers résultats $(10,5 \%$ obtenus par Matsushita sur un module de $1375 \mathrm{~cm}^{2}$ ) soient très encourageants, les problèmes d'environnement associés à l'utilisation du Cadmium empoisonnent les tentatives de développement de cette filière. 
Les alliages à base de séléniure de cuivre indium CuInSe2 ont été étudiés surtout par Boeing, puis par le NREL aux Etats-Unis, Matsushita au Japon et en Europe par Siemens Solar (ex-ARCO) et ZSW à Stuttgart.

Le diséléniure de cuivre et d'indium (CIS) est un matériau composé de type I-III-VI, de structure chalcopyrite très prometteur, puisque le rendement théorique de l'hétérojonction (n)CdS-(p)CuInSe ${ }_{2}$ se situe autour de $25 \%$

Les cellules à base de composés chalcopyrites quaternaires du type $\mathrm{Cu}(\mathrm{Ga}, \mathrm{In})(\mathrm{Se}, \mathrm{S})_{2}$ ont récemment atteint des rendements de conversion record de 18,8\% en laboratoire (NEREL) et malgré les difficultés connues pour maîtriser cette filière à grande échelle, $12,8 \%$ ont été obtenus par ZSW sur $730 \mathrm{~cm}^{2}$.

\section{2) Techniques de dépôt des matériaux du type CIS}

Parmi les très nombreuses méthodes de dépôt essayées, on notera: la co-évaporation des trois éléments, la sélénisation de films d'In et de $\mathrm{Cu}$, l'électrochimie et la technique de pulvérisation par spray. La première méthode est celle qui a permis au NREL d'atteindre les plus hauts rendements. La dernière citée est certainement la plus simple à mettre en oeuvre mais son rendement matière est assez faible.

En raison des problèmes d'environnement suscités par la présence de $\mathrm{Cd}$ dans la couche fenêtre, la communauté scientifique cherche actuellement à le remplacer par des matériaux inoffensifs, tels que certains oxydes transparents semiconducteurs comme le $\mathrm{ZnO}$.

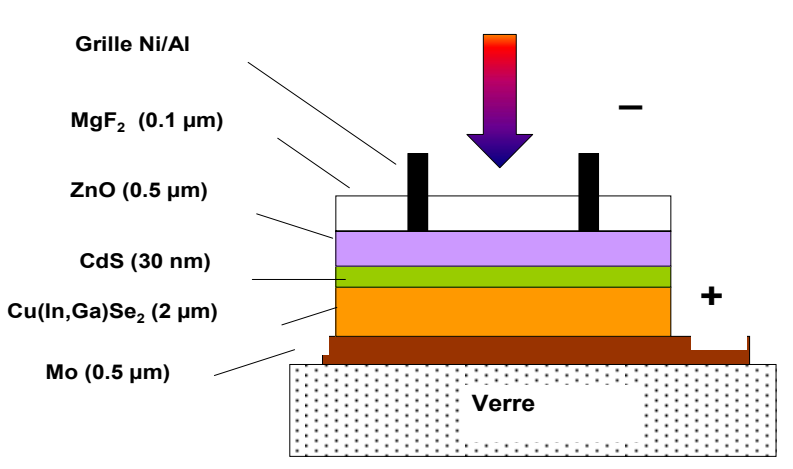

Fig.15. Vue en coupe d'un cellule à base de CuInSe.

\section{3) Applications industrielles}

Une production industrielle a été amorcée par Siemens Solar depuis Juillet 98 puis par Wurth-Solar avec la sortie de modules de $10 \mathrm{Wc}$. Les principales améliorations sont venues de l'élargissement de la bande interdite du CuInSe
$\left(\mathrm{E}_{\mathrm{G}}=1.02 \mathrm{eV}\right)$ par l'utilisation d'alliages du type $\mathrm{CuGaSe}_{2}$ et $\mathrm{CuInS}_{2}$. Le coefficient d'absorption est 100 à 1000 fois plus fort que celui du silicium cristallin dans la gamme 1.1 à $2.6 \mathrm{eV}$.

En ce qui concerne la filière électrochimique un projet nommé CISEL financé par l'ADEME associe un laboratoire du CNRS (LECA) à EDF [21]. Le but du laboratoire commun CNRS-EDF (IRDEP) installée à Chatou est de faire passé cette filière du stade du laboratoire au stade industriel en réalisant des modules de $30 \times 30 \mathrm{~cm}$.

\section{4) Perspectives}

Les films minces de Si micro-cristallin et de CuInSe2 sont des outsiders sérieux, capables en principe de rivaliser avec les performances combinées de rendement et de coût du Si cristallin massif. Leur développement, comme pour les nouvelles cellules $\mu$-morphes, passera par un travail de recherche relativement long avant d'envisager une industrialisation substantielle probablement au-delà de 2010 .

\section{Matériaux en couches minces du futur}

La production d'énergie électrique utilisant des matériaux organiques et/ou nano-structurés semiconducteurs reste un défi technologique majeur pour les prochaines années.

\section{1) Etat de l'art: matériaux organiques}

Les cellules à base de matériaux organiques ont de fait deux atouts majeurs, un coût de production des produits faible et une mise en forme relativement facile. Les enjeux économiques sont évidents et l'utilisation de ces types de matériaux pour la préparation de dispositifs photovoltaïques plastiques est un thème de recherche en plein essor.

Néanmoins, l'efficacité de ces systèmes pour la conversion de l'énergie lumineuse en énergie électrique reste pour le moment modeste (record de rendement de 3,5 $\%$ longtemps détenu par l'Université de Linz en Autriche avec une stabilité ne dépassant pas 500 heures [22], depuis d'autres équipes ont annoncé 5 et $5,7 \%$. En France un projet CSPVP [23] associant le CEA et le CNRS à permis d'obtenir rapidement un rendement de $2,9 \%$ sur ce même type de matériaux.

\section{2) Synthèse et réalisation des structures}

Les cellules solaires photovoltaïques plastiques sont couramment réalisées à partir de la synthèse de petites molécules (jonction P-N) ou d'un mélanges de polymères (réseaux interpénétrés). 


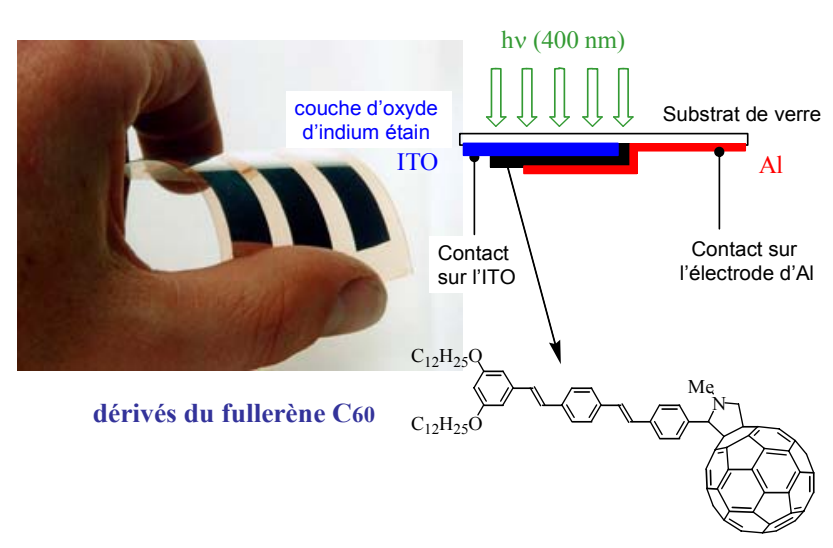

Fig.16. Vue en coupe d'une cellule souple à base de matériaux organiques dérivés des fullerènes [22].

\section{3) Perspectives : matériaux nano-structurés}

De nouvelles voies exploratoires sont engagées qui portent par exemple sur l'intégration de nanocristallites (minéraux ou organiques) dans différentes couches fonctionnalisées pour une conversion par luminescence de photons UV (trop énergétiques) en photons dans le domaine du visible.

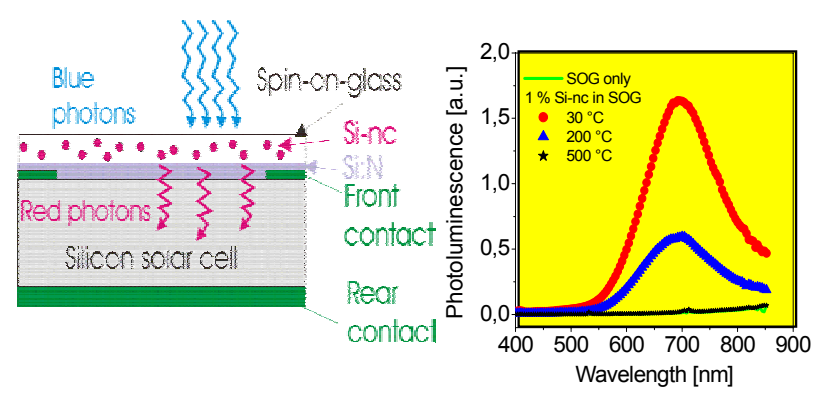

Fig.17. Concept de cellule à couche fonctionnalisée contenant des nanoparticules de silicium [24]

\section{MARCHE DU PV}

La production industrielle mondiale de modules photovoltaïques progresse de $35 \%$ en moyenne depuis presque 10 ans et a franchi le seuil des 2 GWc en 2006 avec un total cumulé confirmé pour 2005 de 3,7 GWc [2].

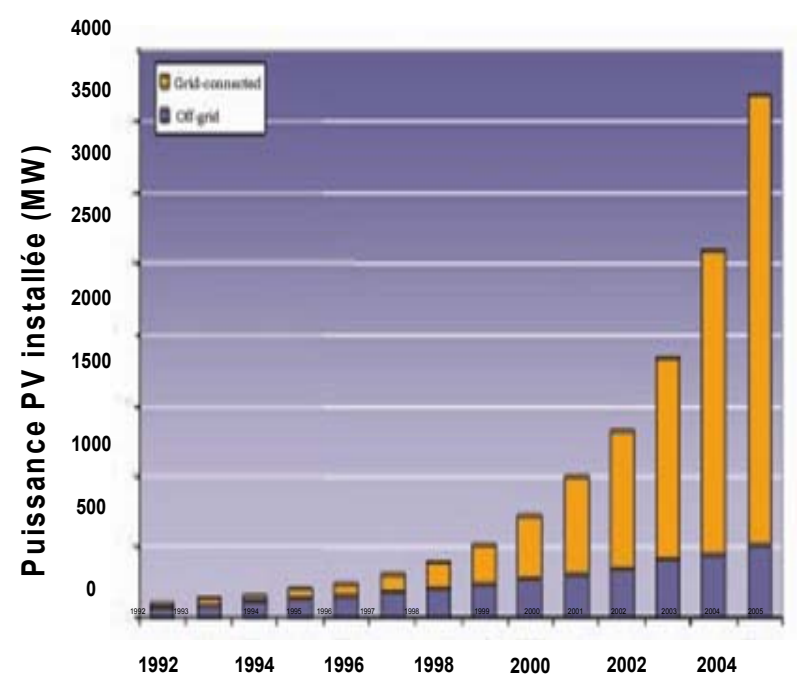

Fig. 18. .Puissance mondiale cumulée du photovoltaïque fin 2005 , montrant la prépondérance du connecté au réseau par rapport aux sites isolés [2].

Le prix de revient du Watt photovoltaïque a également considérablement baissé, il est aujourd'hui tombé aux environs de $2 €$ pour les modules PV (avec un prix de vente de 2,6€, en baisse constante de 10 à 15 centimes d'euro par an) et un coût de l'ordre de 5-6 $€$ pour un système (de l'ordre de $3 \mathrm{KW}$ ) connecté à un réseau [25] et de 8 à $10 €$ pour un système en site isolé avec stockage part batteries.

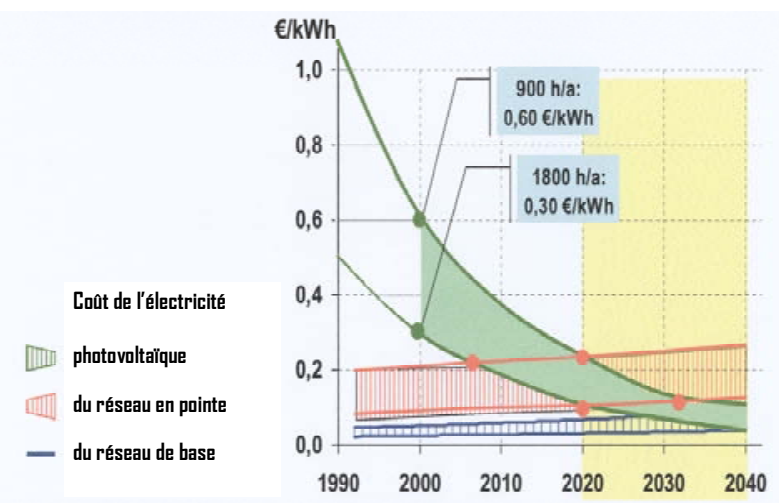

Fig. 19. .Evolution du prix du kWh PV [26].

Le coût du kWh produit qui est estimé dans la fourchette de 30 centimes d'euro pour l'Europe du Sud et à 60 centimes pour l'Europe du Nord (voir figure 19) [26]. Son coût continuera de baisser pour rentrer dans la gamme de prix de l'électricité de pointe dès 2020 et de base en 2040 . 
La part de l'électricité photovoltaïque dans la production mondiale sort du bruit de fond et peut enfin être inscrite $(0,03 \%$ de la production mondiale). Les installations se partagent entre des sites isolés d'une part (habitat isolé, pompage de l'eau, balises maritimes, relais téléphoniques, etc...) et d'autre part connectées au réseau (centrales et surtout les toits photovoltaïques).
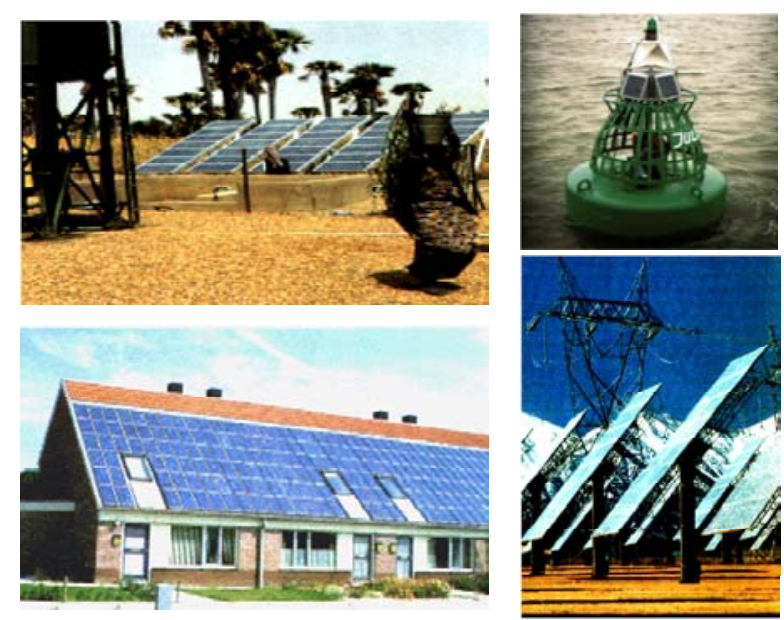

Fig. 20. Les quatre grand secteurs d'application du photovoltaïque : deux exemples en sites isolés (pompage de l'eau et balises maritimes) et en connecté au réseau (toits photovoltaïques, et centrale).

Ce dernier segment de marché ne cesse de croître de sorte qu'à l'horizon 2020 le photovoltaïque pourrait représenter prés de $1 \%$ de la consommation mondiale d'électricité et plus de $14 \%$ à l'horizon 2040 c'est à dire plus que les prévisions pour la production d'électricité d'origine nucléaire.

\section{ANALYSE DU CYCLE DE VIE}

Les dispositifs réalisés à partir de matériaux à base de silicium cristallin et multicristallin (c-Si et mc-Si) restent sans conteste les plus stables avec une garantie de durée de vie donnée par les industriels pour le module PV de 25 ans (avec une perte de rendement inférieure à $20 \%$ ) et à l'horizon 2030 cette garantie passera à 40 ans de sorte que le segment phare d'application restera les centrales et pour l'habitat les toitures.

Les films minces à base de Si cristallisés sur des substrats étranger ou transférés sur un verre offrent le même potentiel de durée de vie sauf pour les matériaux amorphes dont la dégradation est serte connue et maîtrisée mais pour lesquels aucun industriel ne s'engage à donner une garantie même de moins de 10 ans, cependant l'obtention de couches semitransparentes sont un atout majeur pour toutes les applications en vitrage.

En ce qui concerne les matériaux en couches minces à base de chalcogénures (CIS, ...) la stabilité est acquise au niveau du laboratoire et l'industrie qui est encore à ses débuts n'a pas assez de recul pour offrir les mêmes garanties que pour le c-Si.

Enfin pour les matériaux polymères ou organiques dont les problèmes de stabilité sont loin d'être résolus, avec moins de 1000 heures sous un éclairement solaire normal, les premiers dispositifs doivent viser les applications intérieures sous faible éclairement pour tout le secteur à ne pas négliger de la domotique et de l'amélioration du confort de vie (codes barre intelligents, transmission d'information et de données, .....)

Du point de vue de l'émission de $\mathrm{CO}_{2}$ liée à l'industrie du $\mathrm{PV}$, se sont les phases de purification du silicium métallurgique qui sont les plus néfastes avec de l'ordre de 30 à 50 gramme de $\mathrm{CO}_{2}$ par $\mathrm{kWh}$ d'électricité $\mathrm{PV}$ produite suivie par la phase de cristallisation des lingots de sorte que le $\mathrm{kWh} \mathrm{PV}$ contribue pour 60 à $100 \mathrm{~g}$ de $\mathrm{CO}_{2}$ pour tout son cycle de vie (10 fois moins que les énergie fossiles mais 10 fois plus que l'éolien) avec un temps de retour sur énergie de l'ordre de $20 \%$ de sa durée de vie garantie.

Afin de réduire cet impact, les recherches autour du développement d'un matériau de qualité solaire avec des techniques de purification simplifiées, vers des croissances en continu des lingots, vers des plaquettes sciées de plus en plus minces, des rubans et bien sûr vers toutes filières en couches minces vont dans ce sens (pour plus de détail se référer à l'ouvrage [27]).

\section{CONCLUSIONS \& PERSPECTIVES}

Comme nous l'avons mentionné, les matériaux à base de silicium cristallin évoluent sans cesse, vers des économies de plus en plus importantes de matière première, vers des film minces de Si cristallisés sur des substrats étranger ou transférés par pelage sur un verre ou un plastique avec un énorme potentiel à explorer dans le domaine des matériaux nanocristallins afin que cette filière reste compétitive dans les vingt ans à venir avec les matériaux en couches minces à base de chalcogénures (CIS, ...) et bien plus tardivement avec les matériaux organiques.

En effet, c'est à ces horizons que la plupart des experts entrevoient une production industrielle significative à base de films minces de CIS avant l'émergence de nouveaux concepts de cellules à base de matériaux polymères ou organiques (si les problèmes de stabilité peuvent être résolus). 
Pour d'autres experts, dont le professeur T. Saitoh, pionnier du silicium cristallin au Japon, la filière à base de silicium cristallin sera " éternelle ». Le développement des billes de silicium (de $0,6 \mathrm{~mm}$ de diamètre) imprimées sur un substrat souple d'aluminium (Photowatt Int., Canada) est un bon exemple des capacités du silicium à rebondir dès l'émergence de technologies concurrentes.

\section{BIBLIOGRAPHIE}

\section{Jean-Claude MULLER, 59 ans}

Ingénieur de Recherches CNRS au laboratoire PHASE de Strasbourg depuis 1978, Thèse d'Etat : Cellules Solaires à base de Silicium en 1982. Cristal du CNRS-SPI en 1998, Directeur Adjoint du laboratoire PHASE de 1999 à 2005, Directeur Technique à la création d'InESS en Juin 2005.

Chargé de mission du CNRS-STIC (2002-05) pour l'énergie solaire Photovoltaïque (PV) au Programme Energie du CNRS. Membre du comité d'experts de l'ADEME (depuis 2003). Membre du comité d'évaluation de l'ANR PV (depuis 2005). Membre (depuis 1998) de l'Agence européenne de développement des Energies Renouvelables, EUREC Agency, Bruxelles.

\section{REFERENCES}

[1] A. Goetzberger, J. Luther and G. Willeke, Proc. $12^{\text {th }}$ Int'l PVSEC Conf, Jeju, Korea (2001) p 5.

[2] World Energy Outlook - IEA Annual Report 2006

[3] N. Yuge et all, $11^{\text {th }}$ Intern. Photovoltaic Science \& Engineering Conf. Sept 20-24, 1999, Sapporo, Hokkaido, Japan, p 115.

[4] M.A. Green, A. Wang, G.F. Zheng, Z. Zhang, S.R. Wenham, J. Zhao, Z. Shi, C.B. Honsberg, Proc. 12th EC PVSEC Amsterdam (1994) p. 776.

[5] J. Fally, D. Guignot, L. Goeffron, Proc. 7th EC PV SEC Sevilla (1986) p. 754.

[6] B.H. Authier, German Patent (DOS) No. 250883 (1975); H. Fischer, Proc. 1rst EC PV SEC Luxembourg (1977) p. 52.

[7] PHOTOWATT International S.A., FR-38300 Bourgoin-Jallieu.

[8] K. Kaneko, T. Misawa, K. Tabata, Proc. 21rst IEEE PVSC Kissimmee (1981) p. 674

[9] I. Perichaud, G. Dour, F. Durand, D. Sarti, G. Goaer, Q.N. Le, F. Floret, S. Martinuzzi, Proc. 13th EC PV SEC Nice (1995).

[10] EMIX S.A., 23300 La Souterraine (France).

[11] A. Rohatgi, V. Yelundur, J. Jeong, A. Ristow, A. Ebong, $10^{\text {th }}$ Workshop on crystalline Silicon Solar
Cell Materials and Process, August 2000 (CO), p 12

[12] R.G. Seidensticker, L. Scudder and H.W. Brandhorst, Proc. 11th IEEE PVSC Scottsdale (AZ) (1975) p. 299

[13] R.B. Bergmann, Appl. Phys.A 69, 187 (1999).

[14] A. Slaoui et P. Siffert, "Polycrystalline Silicon Films for Electronic Devices" in Silicon: Evolution and Future of a Technology, Eds by P. Siffert \& E. Krimmel, Springer-Verlag, Berlin-Heidelberg (2004) pp 49-72

[15] R.B. Bergmann, T.J. Rinke, C. Berge, J. Schmidt and J.H. Werner, Proc. $12^{\text {th }}$ Int'l PVSEC Conf, Jeju, Korea (2001) p 29.

[16] P.J. Riberon, A. Beaumont, A. laugier, A. Kaminski, A. Fave, M. Lemiti, G. Fantosi, ADEME-CNRS Annual Seminar on PV materials and processes, Sophia-Antipolis 2002 p 111

[17] Sté SOLEMS, Palaiseau (France).

[18] A. V. Kharchenko, V. Suendo, and P. Roca i Cabarrocas: "Plasma studies under polymorphous silicon deposition conditions". Thin Solid Films

[19] Sawada Toru et al, High Efficiency a-Si/c-Si Heterojunction solar Cell, 1st WCPEC (Hawaï, 1994), p 1219.

[20] M. Taguchi et al, "HIT Cells-High Efficiency crystalline Si cells with Novel Structure", Prog. Photovolt: Res. Appl. 8, 503 (2000).

[21] J.F. Guillemoles et al, ADEME-CNRS Annual Seminar on PV materials and processes, SophiaAntipolis 2003 p 45

[22] S.E. Shaheen, C.J. Brabec, N.S. Sariciftci, F. Padinger, T. Fromherz, J.C. Hummelen, Appl. Phys. Lett. 78, 841-843 (2001).

[23] C. Sentein, ADEME-CNRS Annual Seminar on $\mathrm{PV}$ materials and processes, Sophia-Antipolis 2003 p 71-78

[24] V. Švrcek, A. Slaoui, J-C Muller, Silicon nanocrystals as light converter for solar cells, Thin-Solid-Films, 451-452, 384-388 (2004).

[25] Eurec Position Paper, Eurec Agency, BE-1040 Bruxelles, Ed.2000.

[26] EPIA : Présentation de l'étude du Groupe «PV Track » avec les industriels d'EPIA - A Vision for PV Technology up to 2030 and beyond - EC Meeting: Future Vision of PV, Brussels, 28 Sept.(2004).

[27] J.-C. Muller et P. Siffert, "Silicon for Photovoltaics" in Silicon: Evolution and Future of a Technology, Eds by P. Siffert \& E. Krimmel, Springer-Verlag, Berlin-Heidelberg (2004) pp 7392. 